\title{
Tres historias convertidas en leyendas patrimoniales del Chocó Biogeográfico: Nóvita (Nóvita Viejo), Istmina (Negría) y Tadó (EI Carmelo)
}

\section{Three histories turned into patrimonial legend of the Chocó Biogeográfico: Nóvita (Nóvita Viejo), Istmina (Negría), Tadó (El Carmelo)}

\author{
Luz América Lozano*
}

\section{RESUMEN}

Por la escasa información disponible proveniente de investigaciones que permitan conocer los cambios que se han suscitado por circunstancias del tiempo en Nóvita (Nóvita Viejo), Istmina (Negría), Tadó (El Carmelo), este estudio propendió por confrontar el panorama histórico de los distintos acontecimientos que la explotación ejercida por los españoles utilizando esclavos traídos de África, generó sobre los recursos mineros y medio ambientales de esta región. Se levantó la información en campo mediante la metodología IAP (Investigación, Acción, Participativa), complementada con la elaboración de encuestas y criterios personales, Obteniendo entre otros propósitos, desentrañar las riquezas y los valores antropológicos, arqueológicos, sociales y culturales que poseen las zonas estudiadas, finalmente se puede afirmar que a través de la esclavitud, los españoles contribuyeron de manera inconsciente a crear un patrimonio material e inmaterial, que estamos obligados a rescatar, reconstruir y elevar como parte identitaria del pueblo chocoano.

Palabras clave: Esclavitud; Patrimonio; Tradición; Extracción minera.

\author{
Antropóloga, Investigadora principal Com- \\ ponente Sociocultural, Instituto de Investi- \\ gaciones Ambientales del Pacífico (IIAP); \\ Quibdó, Chocó. \\ e-mail: americosa29@yahoo.es \\ Recibido: enero 15, 2009 \\ Aceptado: febrero 2, 2009
}

\begin{abstract}
Due to the little information available, originating of investigations, that allow to know the changes that have been provoked by circumstances of the time in Nóvita (Nóvita Viejo), Istmina (Negría), Tadó(El Carmelo), this study inclined to confront the historical panorama of the different events that the operation exerted by the brought Spaniards using enslaved of Africa, generated on environmental the mining resources and average of this region. By means of methodology I.A.P. (Investigation, Action, Participating), complemented with the elaboration of surveys and personal criteria; the information in field rose. Obtaining among others intentions, to unravel the wealth and anthropological, archaeological, social and cultural the values that own the studied zones, finally can be affirmed that through the slavery the Spaniards, contributed of unconscious way to create material and immaterial a patrimony, who we are forced to rescue, to reconstruct and to elevate like identity part of the chocoano town.
\end{abstract}

Keywords: Slavery; Patrimony; Tradition; Mining Eextraction. 


\section{INTRODUCCIÓN}

Según investigaciones realizadas inicialmente por el etnólogo Velázquez $(1957,1961)$ se afirma que el conocimiento de la medicina tradicional en el departamento del Chocó, es una de las más grandes fortalezas de sus comunidades, mostrándolo como un patrimonio del conocimiento ancestral, que debe ser rescatado.

En cuanto a los metales preciosos, West (1972) es uno de los pioneros de las investigaciones relacionadas con la explotación minera en el departamento del Chocó y elaboró un panorama histórico de este proceso desde los tiempos de la colonia hasta llegar a los procesos de explotación tecnificada impuestas por los gringos, que se evidencia en los pueblos objeto de esta investigación.

Melo (1975) hace una descripción de cómo se ha maniobrado el proceso de explotación minera en Colombia, haciendo mayor énfasis en las distintas políticas de aplicación en los pueblos del departamento del Chocó. Por otro lado Leudo (2009), describe las circunstancias históricas y distintos aconteceres del proceso de explotación, sobre todo en el corregimiento de Nóvita Viejo, municipio de San Gerónimo de Nóvita.

El proceso de revisión literaria demostró que es escasa la información producto de investigaciones que permita conocer los cambios que se han suscitado por circunstancias del tiempo en Nóvita (Nóvita Viejo), Istmina (Negría), Tadó (El Carmelo), como también por la falta, tanto de políticas gubernativas y voluntad administrativa, que pudiese convertirse en apoyo y soporte al desarrollo de estas comunidades.

Esta investigación busca, confrontar el panorama histórico de los distintos acontecimientos que la explotación ejercida por los españoles utilizando esclavos traídos de África, generó sobre los recursos mineros y medio ambientales de esta región. Rescatar en lo posible las riquezas patrimoniales tangibles e intangibles de las comunidades investigadas, de modo que todos los saberes y el conocimiento ancestral del hombre chocoano y su riqueza material, pueda ser promocionada y puesta al servicio de la humanidad.

\section{ÁREA DE ESTUDIO}

Nóvita es un municipio colombiano ubicado en el departamento de Chocó, fundado en 1709. Inicialmente el caserío estaba a orillas del río Tamána y se trasladó a la quebrada Nóvita en 1709 por la abundancia de oro. En 1739 se formó la Provincia del Chocó reuniendo las tenencias de Nóvita, Quibdó y Baudó, designándose a Nóvita como su capital y en 1854 se le trasladó al lugar que hoy ocupa con el nombre de San Jerónimo de Nóvita. Se localiza a 132 km de la capital del departamento, a una altura de $70 \mathrm{~m}$ y una temperatura promedio de $28^{0} \mathrm{C}$.
Su economía se basa en actividades como la agricultura (plátano, arroz, maíz, yuca y frutas), la minería (plata, oro y platino) y el comercio de algunos de estos productos. Cuenta con los corregimientos de Cajón, Carmen de Suramá, Irabubú, La Playita, San Lorenzo, Sesego, El Tigre y Urabará; su población aproximada es de 7867 habitantes entre afrodescendientes e indígenas primordialmente.

Istmina es un municipio de Colombia ubicado en el departamento de Chocó, fundado en 1834 por el señor Juan Nepomuceno Mosquera, inicialmente con el nombre de San Pablo, el cual fue cambiado en 1903 por el nombre actual. Su extensión es de 2480 kilómetros cuadrados y cuenta con una temperatura promedio de $25.9^{\circ} \mathrm{C}$. Se encuentra a 75 kilómetros de Quibdó y a 79 metros sobre el nivel del mar. La palabra Istmina se forma de la contracción de las palabras Istmo y Mina, dos características del área en el cual fue fundada.

Cuenta con los corregimientos de Dipurdú, Noanamá, Paitó y Salazar entre otros; tiene 11 inspecciones de policía, 2 resguardos indígenas y 15 caseríos. Su población alcanza los 42.381 habitantes. La economía está basada en la agricultura, la pesca y la minería como en la mayoría de los pueblos del Chocó.

Tadó comienza su vida administrativa desde 1821 con la Ley 8, que designa a Tadó como Distrito del Cantón del San Juan, perteneciente a la Provincia del Chocó, la cual estaba incorporada al Estado del Cauca. En 1857, por la Ley suscrita de la Presidencia, Tadó se constituye en el Círculo Electoral $\mathrm{N}^{\circ} 31$ con un total de 6.388 habitantes. En 1906, antes de dividirse la Intendencia del Chocó en las dos provincias del San Juan y el Atrato, Pueblo Rico deja de ser corregimiento de Tadó y se constituye en municipio del departamento del Chocó.

El municipio de Tadó está ubicado en la parte oriental del departamento del Chocó, dentro de la zona del Alto San Juan, con un área aproximada de $878 \mathrm{~km}^{2}$ y a $66 \mathrm{~km}$ de la ciudad de Quibdó; su cabecera municipal esta ubicada a los 76³'10" de longitud occidental, al margen izquierdo del río San Juan y a la derecha del río Mungarrá.

Limita por el norte con los municipios de Certegui y Bagadó, por el sur con el municipio de Rio Iró, al occidente con Unión Panamericana y al oriente con el departamento de Risaralda. Se localiza a $75 \mathrm{msnm}$, con una temperatura media entre $27^{\circ} \mathrm{C}$ y $28^{\circ} \mathrm{C}$.

La economía de este municipio se basa en la agricultura y la minería, predominando el cultivo del plátano, chontaduro, borojó, piña, yuca, banano, caña, marañón, lulo, anón, cacao, papaya, mil peso y de maderables como guasca, carbonero, caucho, chano, laurel, trúntago y guayacán, trabajada con procesos rudimentarios, lo que permite mayor desarrollo en el sector. Con respecto a la minería, los suelos son ricos en oro y platino, que son extraídos por la gente de manera artesanal con la técnica conocida como bareque, aunque en la actuali- 


\section{Bioetnia Volumen 6 № 1 (enero-junio), 2009}

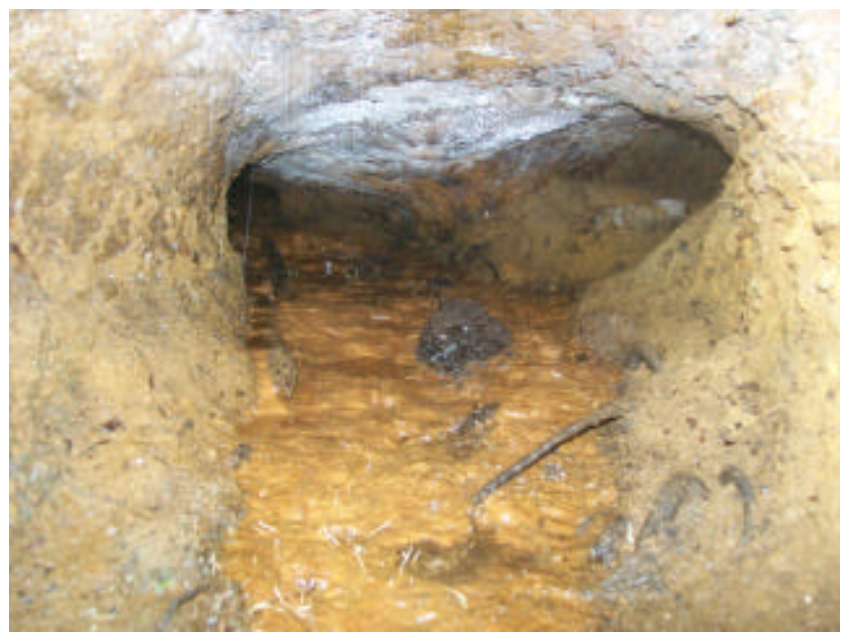

Figura 1. Socavones, elaborados por los esclavos, por donde se realizaba la extracción minera en Nóvita Viejo

dad se ha visto invadida la zona por los medianos empresarios que utilizan maquinaria pesada como la retroexcavadora. De ahí que uno de los grandes problemas ecológicos del municipio se presente por la falta de compromiso de este tipo de empresarios, porque no realizan actividades de llenado y readecuación de los terrenos, dejándolos no aptos para la siembra, acabando la capa vegetal y formando posos retenedores de aguas estancadas, incrementando así la cría de vectores (zancudo) trasmisores de distintas enfermedades. La explotación de recursos forestales se realiza de manera irracional, permitiendo la pérdida de recursos económicos para el municipio y para los dueños de los terrenos donde se realiza esta práctica.

\section{MÉTODOS}

La información que se presenta fue obtenida utilizando la metodología IAP complementada con la elaboración de encuestas, que se aplicaron a distintas personalidades de Nóvita (Nóvita Viejo), Istmina (Negría), Tadó (El Carmelo), con edades que oscilaban entre los 18 y 96 años de edad.

\section{RESULTADOS Y DISCUSIÓN}

En Nóvita Viejo se logró auscultar los distintos vestigios que hoy constituyen patrimonio y evidencia del sometimiento de los esclavos en los socavones (Figura 1). Igualmente, se pudo conocer lo que se determinó como el Tribunal del Juicio, en donde se juzgaban a los esclavos que no cumplían con el establecimiento de Ley demandado por la Corte Española. De igual manera, se logró llegar a lo que se constituyó como el Panteón de los Muertos, en donde se sacrificaban a los esclavos que supuestamente no cumplían la misión encomendada.

Pero, Nóvita Viejo muestra también los socavones donde existió el peor de los procesos ejecutados por los españoles en su empresa explotativa: eliminar a los esclavos a través de la guillotina cercenándoles la cabeza por el supuesto de haber perdido o apropiarse de un mínimo adarme de la riqueza obtenida.

Estas son expresiones manifiestas por los distintos acompañantes, quienes condujeron a verificar sus diferentes versiones in situ, constatar que los procesos históricos acontecidos en Nóvita y Nóvita Viejo, puedan ser considerados en la posibilidad de la construcción real de la historia colombiana, para que ameriten ser tenidos en cuenta como patrimonio de la justicia de los pueblos dominados.

Se pudo constatar también que Nóvita es uno de los pueblos más emblemáticos que posee el departamento del Chocó, si tenemos en cuenta que ha sido capital de este territorio. Si reconocemos que después del Canal del Cura, fue el objetivo principal de la explotación minera por parte de los españoles y en donde se gestaron quizás los más grandes y graves acontecimientos de la sumisión de vida y muerte de los esclavos africanos proferidos por los españoles. En este pueblo están las evidencias que permitirían reconstruir la fatal historia que engrandeció el corazón de los explotadores y adormeció con nostalgia, pesar y dolor el corazón de los negros explotados y sometidos a la más cruel barbaridad que la historia del país y del mundo, pueda recordar como la esclavitud.

Nóvita es también, cuna de hidalguía si tenemos en cuenta que fue allí el natalicio de los quizás hombres más notables del departamento del Chocó, como lo fueron los hermanos Carlos y Jorge Holguín Mallarino, de quienes su prestigio, prestancia e intelectualidad permitió dirigir los destinos del país como presidente de la república tras la inteligencia y capacidad del mayor de ellos, Carlos Holguín. No desconocemos tantas personalidades ilustres que ha tenido y tiene el Chocó, pero ningún pueblo de esta región de la patria, ha tenido como Nóvita un hijo suyo que haya dirigido los destinos del país, tras el solio de Bolívar. Estos pequeños detalles, elementos naturales y materiales, se constituyen en indicadores tangibles e intangibles que simbolizan y reflejan una riqueza patrimonial que amerita el reconocimiento de las autoridades de todo orden cultural, pues permiten rescatar las riquezas y las grandezas del Chocó biogeográfico, pluriétnico y multicultural.

En Negría, corregimiento del municipio de Istmina, ubicado a orillas del río San Juan, el proceso de explotación colonizadora de los peninsulares (venidos de España), en las riveras del río San Juan convirtieron a esta pequeña población en un centro de abastecimiento, donde se almacenaban las provisiones para el sostenimiento de las distintas cuadrillas 


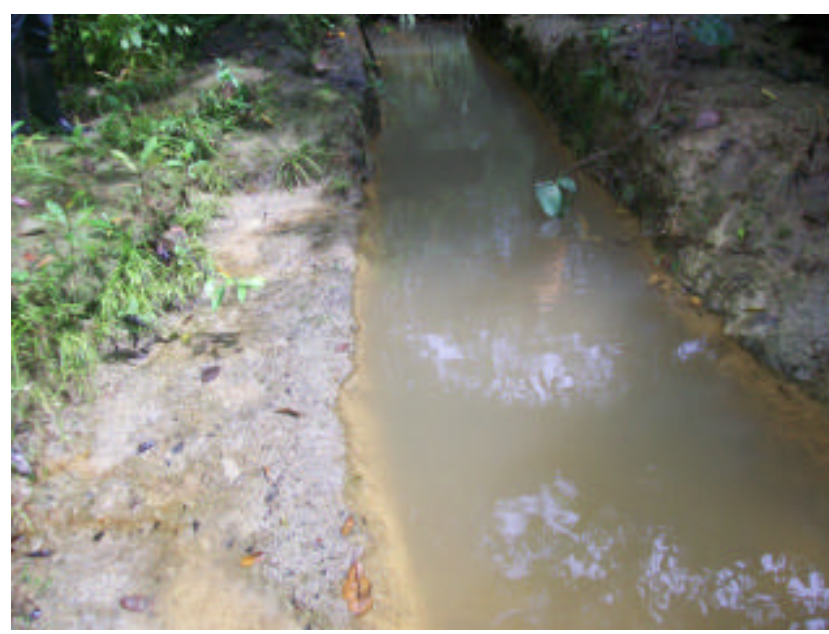

Figura 2. Yacimiento aurífero construido en Negría (I stmina) por los esclavos

de esclavos que explotaban los socavones y los yacimientos auríferos (Figura 2).

Este corregimiento se constituyó en una reserva estratégica por su ubicación geográfica de donde se facilitaba el desempeño minero y era punto expedito para continuar con las comisiones de conquista y explotación de otros lugares de la cuenca del río San Juan como es el caso del hoy municipio de Sipí.

Negría es un referente histórico de conservación patrimonial en donde se puede constatar los distintos vestigios de grandeza y señorío que quedaron como testimonio ático del laboreo minero que permitió cambiar el cauce de su quebrada principal, con el esfuerzo esclavo para derramar sus aguas al río San Juan.

Uno de los más grandes elementos patrimoniales encontrados en esta reserva ecológica, es la riqueza forestal ubicada en uno de los enclaves más cerrados y bravíos de la selva chocoana (Figura 3 ).

Pero uno de los más grandes patrimonios inmateriales existentes en el corregimiento de Negría que deben ser rescatados, son los que se relacionan con las prácticas y rituales que evidencian concepciones sobre la vida y la muerte que se remontan al legado africano y a la inusitada capacidad de creación cultural de todos sus habitantes.

Dentro de estas prácticas y rituales es pertinente resaltar aquellas relacionadas con la medicina tradicional y los rituales fúnebres donde se cantan los alabaos, que mediante la interrelación que hoy existe con los indígenas, crean intercambio cultural que antes no se producía participando estos, con el canto del jai en la procura de la sanación de la salud (Figura 4.)

Es así como se puede determinar una riqueza patrimonial inmaterial de urgente rescate asentada en la sabiduría de los amerindios y afrodescendientes chocoanos, que puede de-

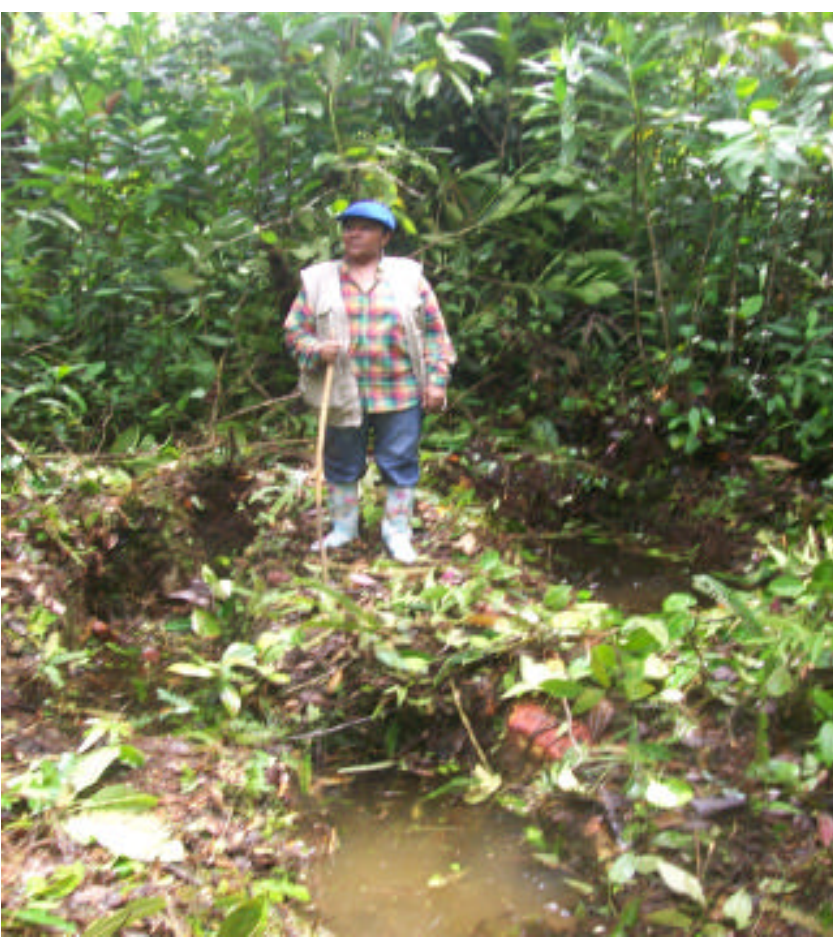

Figura 3. Regenaración vegetal de los antiguos entables mineros de Negría (I stmina)

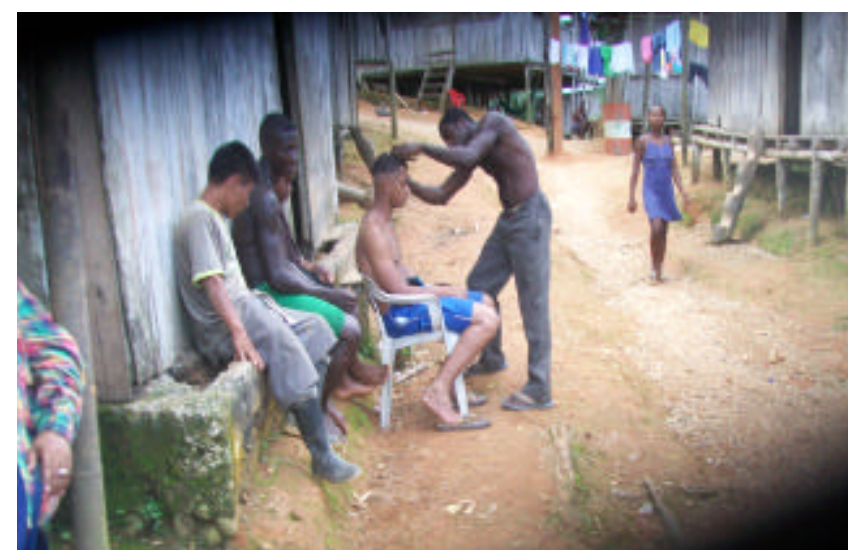

Figura 4. Relación intercambio cultural entre indígenas y afrodescendientes en Negría (I stmina)

nominarse como un conglomerado de conocimientos y técnicas de intervención sobre la enfermedad y el daño, que se asocian estrechamente con la cosmovisión de sus pobladores.

Estas circunstancias muestran a Negría como un emporio de subsistencia cultural antrópica que devela la tesis antropológica, que el devenir de la explotación a que fue sometido el Chocó por parte de los españoles también permitió la creación de iniciativas de supervivencia de sus habitan- 


\section{Bioetnia Volumen 6 № 1 (enero-junio), 2009}

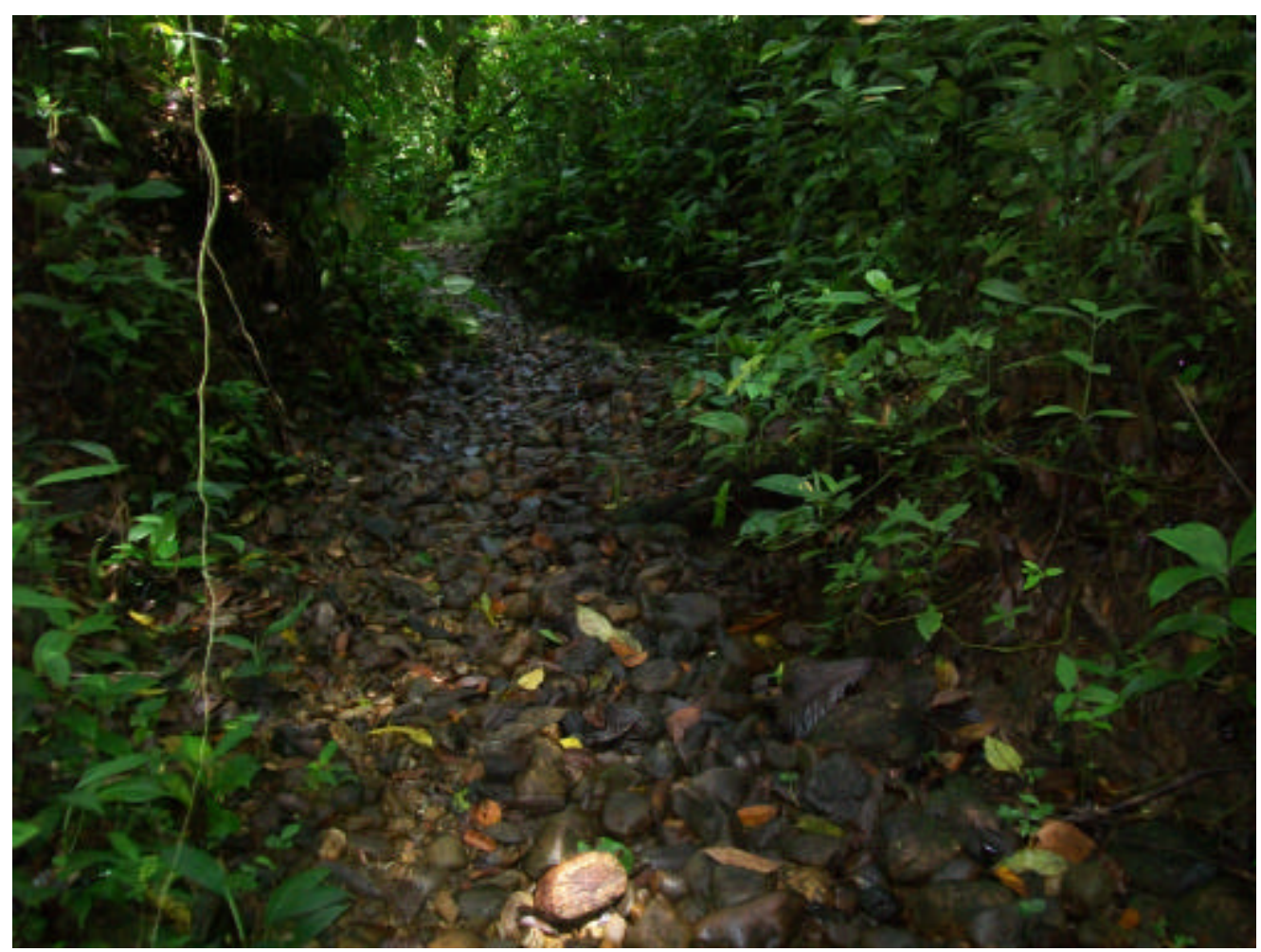

Figura 5. Vestigios de los procesos de explotación medioambiental de los españoles en el corregimiento de El Carmelo (Tadó)

tes a través del esfuerzo material, pero también del desarrollo del conocimiento medioambiental para la preservación de su propia existencia, sin el patrimonio de los esclavizadores. Pero es que la vida patrimonial del Chocó es tan grande, rica y valiosa, que el objetivo del propósito investigativo de este artículo, obligó que mi periplo motivado por los valores antropológicos, arqueológicos, sociales y culturales como los encontrados en Nóvita y Negría, circunscribiera el cumplimiento de la misión laboral en los territorios del municipio de Tadó y en especial en el corregimiento de El Carmelo, pueblo que a pesar del descuido generalizado de todas las regiones del departamento del Chocó, en su seno entraña valores simbólicos de riqueza material, tangible e intangible de rigor histórico y beneficio cultural para el Chocó el país y la cultura universal.

Por otra parte El Carmelo corregimiento del municipio de Tadó, fue un fuerte de resistencia de negros cimarrones que enfrentaron con reconocido coraje las fuerzas invasoras repeliendo el ímpetu avasallador de españoles que a la manera impositora querían adueñarse de los territorios y explotarlos bajo el sometimiento y las condiciones de esclavitud (Figura 5).

El Carmelo como real de minas, conserva una tradición cultural representada en su oralidad, en el conocimiento ancestral de sus mayores y de un manejo de sus recursos biodiversos que no sólo propician la continuidad de sus vidas (por la ausencia pronta y efectiva de los programas de salud), sino que se ha convertido en sitio o lugar «necesario» para consultar y buscar recursos de sanación y buena suerte de las personas que en su acontecer demandan toda clase de sortilegios y «ayudas» que auspicien las soluciones a problemas considerados insolubles para la «mala suerte o el destino».

Los distintos parajes naturales, las cuencas acuícolas y la abrupta naturaleza de su selva bravía, conforman un panorama que se constituye dentro de la reserva forestal del Chocó, en una reserva de conservación y de protección especial para el beneficio del hombre de la región.

En el corregimiento de ElCarmelo se conservan las liturgias traídas del África, las cuales se combinan y entremezclan con manifestaciones linguiísticas de ancestros remotos que apaciguan los vocablos del idioma impuesto por los esclavizadores, mientras que en el arte musical con instrumentos autóctonos, se reviven las danzas y canciones de recordatorio de un pasado lejano que en ocasiones obliga a la nostalgia, en los velorios y entierros de sus muertos. Mientras que en los momentos alejados de la solemnidad del dolor, se despliegan el bullicio y la alegría festiva del hombre bantú, del negro de Nigeria, del afrodescendiente que ha mesclado su cultura de ancestro con los ribetes penetrantes y contagiosos de la obligada asimilación nacional. 


\section{Tres historias convertidas en leyendas patrimoniales. LA Lozano}

Estas y muchas otras razones permitieron reconocer consensualmente, que el departamento del Chocó frente a la historia del país colombiano, se yergue como un patrimonio tangible e intangible, siempre y cuando las posibilidades de investigar los distintos tipos y arquetipos que en la historia de su existencia han forjado y están forjando su propio patrimonio.

En el corregimiento de El Carmelo se encontró, la riqueza de un patrimonio inmaterial que esta más relacionado con el conocimiento ancestral de la cultura afrodescendiente que se reforza con lo material a través del uso cotidiano de su reserva y todos y cada uno de sus elementos biodiversos y de la cotidianidad con que el medio permite el desarrollo de actividades que diferencian a los hombres de este medio con respecto al quehacer de otros.

Aquí está asentada la medicina ayurvédica, las posibilidades de adivinación, los juros y conjuros de sabios, rezanderos, sobadores, emplastadores, parteras, curanderos de mordeduras de serpientes, saladores, los protectores con amuletos, quienes ven la suerte y predicen las posibilidades de beneficio o maleficio, quienes saben «hacer paradas» para «coger o quitar mujeres», quienes «rezan» las minas y canalones, los hoyos y las cuelgas en la producción minera y en definitiva hacen aparecer lo perdido y condenan o juzgan al malvado.

Este es pues, un patrimonio de sabiduría que aun cuando está irrigado en todo el ámbito geográfico del Chocó, el corregimiento de El Carmelo ha hecho historia en el devenir del municipio de Tadó y el departamento y sin competir con ningún otro pueblo chocoano, se ha ganado merecido privilegio por el reconocimiento de quienes han necesitado de los servicios de sus «sabios»; igualmente han logrado los resultados esperados de manera positiva en una alta proporción.

De aquí se desprende el hecho que se afirme que en las tres localidades chocoanas investigadas, se logró descubrir un acervo cultural y patrimonial, que amerita ser rescatado, conservado y promocionado como riqueza necesaria que posibilita el entendimiento del hombre por el hombre, en aras de reestructurar el patrimonio y la identidad de los pueblos, porque es más lo que nos une que aquello que nos separa.

El Carmelo, alejado de las mejores condiciones que plantea el desarrollo, es un pueblo recóndito y sin muchas expectativas y que guarda en su seno la hidalguía y dignidad de haber parido también hombres ilustres y aportar con la bizarría de sus hijos, a la defensa patriótica del yugo esclavizante. Es por ello, que hoy en día, se convierte en un paso obligado de aquellos que llegan o se van, así como lo fue en la procura de los invasores.

\section{CONCLUSIONES}

El proceso propuesto en los objetivos de la investigación, sobre la caracterización, rescate y restauración del patrimonio histórico, arqueológico, social y cultural de los municipios de Nóvita (Nóvita viejo), Istmina (Negría) y Tadó ( El Carmelo), determinó que en estas distintas regiones la presencia de los colonizadores venidos de España, ejerció una influencia de carácter preponderante. Sin embargo, en Nóvita Viejo se desarrollaron quizás las más grandes actividades de explotación minera, y se ejercieron también los mayores cumplimientos del ejercicio de Ley dictaminados por la colonia española.

Descubrir la vida patrimonial de El Carmelo, en sus distintos parámetros material e inmaterial, cultural, social, arqueológica y antropológica, satisface los referentes históricos del Chocó, para exponerlos como verdadero legado que contribuirá a esclarecer la historia del país.

\section{AGRADECIMIENTOS}

Quiero expresar mis más sinceros agradecimientos a cada uno de mis compañeros con los cuales realicé las salidas a campo, también agradezco a las comunidades de Nóvita, especialmente a Didier Darío Gómez, Director de la Fundación Cultural del Tamaná, por su acompañamiento y colaboración incondicional durante mi estadía en la zona. A las comunidades de El Carmelo (Tadó), Zamira Mosquera «La coja Shakira» y a todos los habitantes de las comunidades de Negría (Istmina) que participaron de una u otra manera para que este trabajo fuera una realidad.

\section{LITERATURA CITADA}

Melo, H. 1975. La maniobra del oro en Colombia. Medellín: Editorial la Pulga. Leudo, M. 2009. Nóvita toda una historia. 300 años. 1709-2009. Quibdó: Banco de la República.

Velásquez-Murillo, R. 1957. La medicina popular en la costa colombiana del Pacífico. Rev Colomb Antropol. 6: 195-241.

Velásquez-Murillo, R. 1961. Ritos de la muerte en el alto y bajo Chocó. Rev Colomb Folklore. II (6): 9-74.

West, R. 1972. La minería de aluvión en Colombia durante el período Colonial. Bogotá: Imprenta Nacional. 